\title{
Design Of Power Transformer Online Monitoring System Based On GPRS
}

\section{Mengjie-Zhang ${ }^{1}$, Shunbin-Hu², Jialong-Sun ${ }^{3}$, Ning Zhang ${ }^{4}$}

${ }^{1}$ College of Mechanical and Electrical Engineering, Agricultural University of Hebei, Hebei Baoding, 071001

${ }^{2}$ Zhangjiakou power supply Bureau, Hebei Zhangjiakou, 075000

${ }^{3}$ Academic Administration, Baoding Radio \& Television University, Hebei Baoding, 071051

\begin{abstract}
This paper uses the design scheme which is based on of the microcontroller of C8051F580 and threephase electric energy metering chip, finished collection, processing and transmitting for the signal of the power of the transformer and the state of the switch. This system takes Server SQL 2008 as the background database, and achieves visiting by the ADO.NET data access technology. The management software of the monitoring master station is responsible for receiving, analyzing and processing, to form the graphics, reports and other types. The database access mode in this paper is based on B/S and C/S. The test results showed that the system worked stably, and realized the functions, which realizes the real-time monitoring of the transformer on operation data, remote data transmission, timely alarming and so on.
\end{abstract}

Keywords: GPRS; Power Transformer; On-line Monitoring

\section{INTRODUCTION}

Distribution transformers in the power system with a large number of covering a wide range of distribution characteristics, which requires online monitoring system to provide real-time, stable data transmission ${ }^{[1]}$.For the present, 3G network technology due to the high tariff is not comprehensive and universal, GPRS relatively low cost,wide signal coverage,stability, and the main station can be maintained 24 of comunication. GPRS wireless communication technology as an online monitoring system communication technology $\mathrm{y}^{[2]}$.

\section{SYSTEM OVERALL STRUCTURE DESIGN}

In this paper, the monitoring system is composed of three parts: the monitoring terminal, the GPRS wireless communication system and the monitoring station. The system structure diagram is shown in Figure 1.

The monitoring terminal realizes the acquisition, processing and transmission of the data signals, such as the three-phase electrical parameters of the transformer, and the switching state.

System uses GPRS communication mode, the monitoring data, switch status and alarm information is transmitted to the monitoring station, the

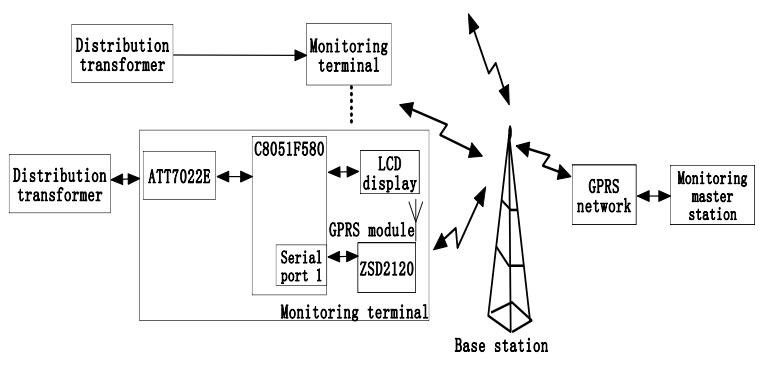

Fig. I The system structure diagram

monitoring station to receive the data information processing analysis and early warning processing.

Monitoring master station system using B/S mode and $\mathrm{C} / \mathrm{S}$ mode combination of architecture $[3,4]$, can be used for multiple power transformers unified management and data acquisition and analysis. Remote users can access to the server on any platform, and meet the needs of different users of the system with different functions.

\section{$2 \quad$ Hardware structure of the system}

The transformer monitoring terminal of the system uses the ATT7022E and C8051 microcontroller combined, and with the watchdog circuit monitoring module, switch input and output module, communication module and other modules, the overall design of the system is shown in Figure 2.

This article is published under the terms of the Creative Commons Attribution License 4.0

Author(s) retain the copyright of this article. Publication rights with Alkhaer Publications.

Published at: http://www.ijsciences.com/pub/issue/2015-12/

DOI: 10.18483/ijSci.892; Online ISSN: 2305-3925; Print ISSN: 2410-4477 


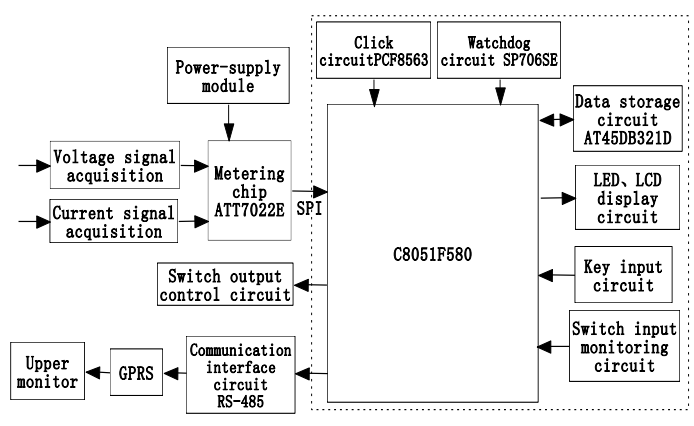

Fig.2 The structure diagram of hardware system

Three-phase current, voltage signal input to the ATT7022E chip,ATT7022E chip for real-time acquisition and calculation of electrical parameters, the results obtained through the SPI interface transmission to the C8051F580 microcontroller,C8051F580 microcontroller set more than the set value alarm or to control, and the control button,the display module can be the operation parameters of the distribution transformer, recording parameters at any time call RS-485 microcontroller interface(Fig. 1 serial port 1) connected with the GPRS module, the monitoring station through GPRS Line communication mode and the monitoring terminal to communicate,so as to achieve remote online monitoring of the running state of the transformer.

\subsection{Power module unit}

Due to the various functional modules of the system and the requirements of the power supply voltage of the chip, the system needs to provide $3.3 \mathrm{~V}, 5 \mathrm{~V}$ and $12 \mathrm{~V}$ three kinds of different voltage magnitude of the power supply.

$220 \mathrm{~V}$ input AC power transformer voltage, rectifier filter to get the $12 \mathrm{~V}$ voltage, $12 \mathrm{~V}$ voltage and then after two LM1085 chip 5V power supply: VCC1 and VCC2.VCC1 are used as the input voltage by LD1117AS33TR $33 \mathrm{~V}$ voltage regulator chip to get 3 $3 \mathrm{~V}$ output voltage, CPU, LED/LCD display circuit, keyboard and other circuit power supply voltage.VCC2 to provide communication circuit and photoelectric isolation circuit, the $9 \mathrm{~V}$ backup battery, ensure the normal operation of the terminal.

\subsection{Data acquisition module}

Three phase current, the voltage input signal acquisition [5] mainly through the mutual inductor and three-phase electric energy measurement chip ATT7022E to complete, the data acquisition circuit as shown in Figure 3.

ATT7022E chip can be used for three-phase three wire line, also can be used for three-phase four wire line. The chip can measure the various voltage

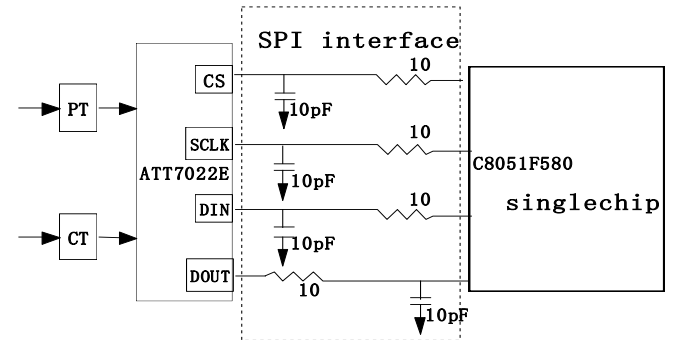

Fig. 3 the block diagram of the data acauisition module

effective value, current effective value, power factor, phase difference and frequency and other parameters, but also can measure the total active power, reactive power, apparent power, active power and reactive power.ATT7022E data access interface [6] (Peripheral Interface SPI), using two control lines and two data lines: CS/SCLK/DIN/DOUT.

In this module, the SPI signal line is connected in series with a small resistor, which is connected with a $10 \mathrm{pF}$ capacitor, which is used to eliminate the oscillation on the SPI interface signal.

\subsection{CPU and peripheral circuit design}

This part is mainly composed of CPU, watchdog unit, data storage unit, clock unit, display unit, communication unit, switch input and output unit.

\subsubsection{CPU}

CPU in this system uses the high performance microprocessor C8051F580, this processor has the rich hardware resources and the fast processing speed.

\subsubsection{Data storage unit circuit design}

Memory using ATMEL's AT45DB321D chip, the storage capacity of $32 \mathrm{Mbit}$, chip power supply voltage is $3.3 \mathrm{~V}$. The chip with serial interface to access data, simplifying the hardware layout, improve the reliability of the system.

\subsubsection{Clock cell circuit design}

Using NXP PCF8563 clock chip, PCF8563 and CPU is connected by bus to achieve all addresses and data transfer, bus maximum transmission rate for the external crystal rate of 400kbit/s. clock chip of $32.768 \mathrm{KHz}$, power supply voltage $3.3 \mathrm{~V}$ and a battery to provide for.

\subsubsection{Display interface circuit design}

In order to allow the field monitoring personnel can see the monitoring of real-time data, the system terminal has a display function. The display can be the operation parameters of the distribution transformer, recording parameters at any time call display. The system in order to simplify the operation, the use of the bus interface design, the use of Epson Corp 
S1D13506 as the display control chip, select TFT (Film Transistor Thin) color display, select V16100 IS41L as the display of memory.

\subsubsection{Watchdog cell circuit design}

This system on the basis of the built-in software watchdog hardware watchdog circuit [7], the selection of the SP706SE company's Exar chip. The chip is a low power, high reliability MCU reset monitor chip. Due to the I/O's MCU port is $3.3 \mathrm{~V}$ power supply, and the chip's working level and capacitance, so they can be directly connected, MCU reset terminal connected with the reset end of the watchdog.

\subsubsection{Communication module}

The communication unit mainly includes RS-232 interface circuit and RS-485 interface circuit, the power supply is provided by the.RS-232 VCC2 interface circuit using MAX232 chip, including two sets of interfaces, respectively, and the field of software communication and field debugging and maintenance of.RS-485 interface circuit using SN65LBC184 chip for monitoring device and GPRS wireless communication module connection.

GPRS wireless communication device using.ZSD2120 module ZSD2120 is a wireless transparent transmission of data using GPRS embedded DTU module, built-in SIEMENS production of industrial grade high reliability GPRS engine (MC35i / MC39i) and high-speed embedded processor. According to the different tasks of sending data, the module is divided into regular transmission, real-time transmission and alarm functions, the received data into character data, and then in accordance with the protocol data frame format to send.

Monitoring data of the monitoring terminal and fault alarm information through a small range of wireless network transmission to give it communication DTU, after the DTU and then through the GPRS network will monitor the information transmitted to the main station.

\subsubsection{Switch input and output module}

The acquisition of the switch status can be realized by collecting the contact position signal. In order to ensure the collection information is correct and reliable, and the interference signal is prevented from entering the CPU, the switch input and the CPU control terminal are electrically isolated from the internal and external circuit by the photoelectric coupler PC817.

\section{Transformer monitoring device software design}

\subsection{Sampling method selection}

The quasi synchronous sampling method is used in this system, which belongs to the periodic sampling and reduces the complexity of the hardware. The system is collected every 10 minutes to the transformer operating parameters and the [8] state information.

\subsection{System software design}

The software flow of the online monitoring and collection system of the transformer is shown in Figure 4.

When a real-time data request, the device through the information to determine whether the occurrence of power outages, lack of phase, the voltage exceeds the upper limit and lower limit, the current limit and transformer outage, switch abnormal action and so on. If the acquisition data is normal, the system will be data storage, while the LCD monitor display monitoring data; if the alarm event occurs, the monitoring software automatic alarm, and the alarm information and related data storage, to achieve the monitoring terminal alarm.

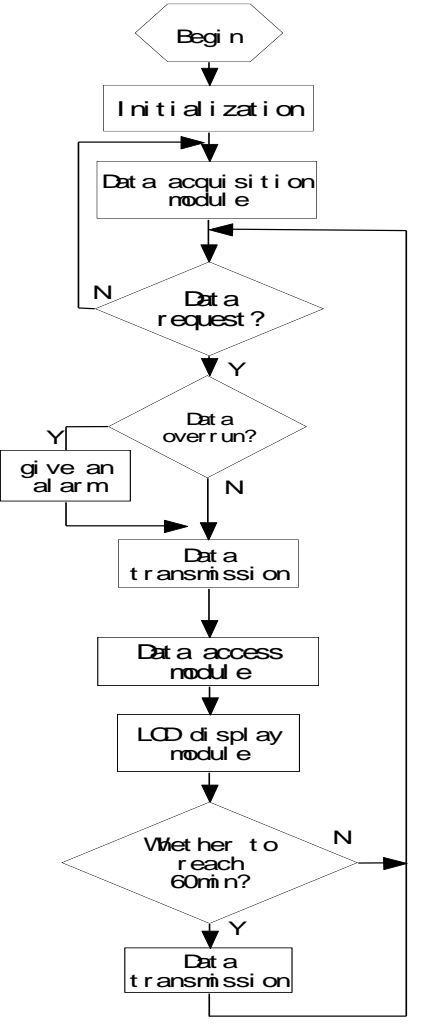

Fig.4 The software flow

diagram
When the system to determine the monitoring time to reach $60 \mathrm{~min}$, monitoring data into the timing of the transmission mode. The system through the DTU terminal of the built-in microprocessor to process the data, the protocol package sent to the wireless network, to achieve through the GPRS network will monitor the timing data to monitor the master station.

Monitoring station to receive the data storage, through the programming to achieve the historical data and state parameters of the query, the parameters and the abnormal state of the alarm limit settings, and generate the corresponding data reports and analysis of the alarm. 
The reasonable database structure can improve the system response speed and improve the integrity and consistency of the data in the system.

\subsection{Database selection}

By comparing with the Office Access Microsoft database, the dynamic real-time monitoring of the transformer requires a higher data storage capacity, and requires a sensitive data storage response, this

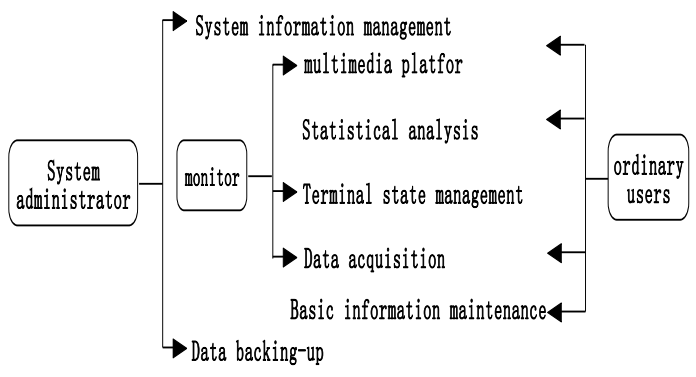

Fig. 5 the illustration of the top level function of the monitoring system

paper uses server SQL to complete the design of the database [9].

\section{$4 \quad$ System architecture model}

Monitoring master station using $\mathrm{C} / \mathrm{S}$ architecture and $\mathrm{B} / \mathrm{S}$ architecture combined with the structure of the software framework. According to the needs of the system business role, is divided into three categories of system administrators, monitoring personnel and ordinary users, the use of two different systems to meet the needs of different users with different permissions on the system, the system top-level function as shown in Figure 5.

\subsection{Implementation of data access}

This article uses the Microsoft.NET framework platform to carry on the system development, uses the ADO.NET data access technology.ADO.NET to be able to use the unified code to process the data, has the disconnected data structure, improves the data access efficiency.

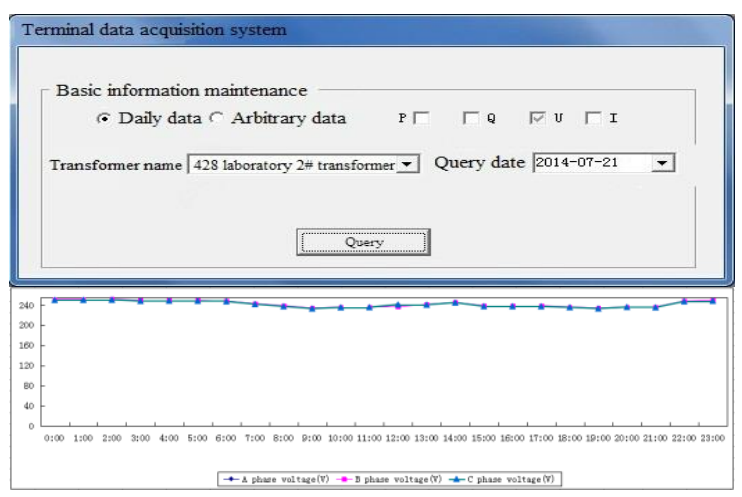

Fig.6 The daily load curve analysis

chart of voltage transformer
5 System display interface

According to the start time and the end time of the system can also be the transformer voltage, current, active power and so do curve analysis, as shown in Figure 6. From the diagram, we can see that the system performance is stable, that is, the system is feasible.

\section{Conclusions}

Through testing, the system runs reliably, using C/S architecture and $\mathrm{B} / \mathrm{S}$ architecture combined with the structure of the software framework, the field monitoring data can be transmitted to the monitoring station in real-time according to the time.

The system completes the data acquisition, the current status and the historical status of the transformer monitoring terminal, with the help of GPRS communication technology, the field distribution transformer terminal data transmission to the master station, the summary to the background system, to achieve real-time monitoring of the transformer's operating parameters.

\section{REFRENCES:}

1. Wang hai-yan, Ye lei. A remote monitoring and control system for distribution transformer based on general packet radio service $[\mathrm{J}]$. Journal Of Shanghai University Of Electric Power, 2011; 27(1): 57-60.

2. Sun lei, Bao yi-qin. Design and implementation of distributed transformer monitoring system[J]. Electrical Measurement \& Instrumentation, 2008, 45(4):36-39.

3. Zhang zhen-wei. The study of remote supervisory control and data acquisition of distribution transformer based on gprs[D]. Chendu: Southwest Jiaotong Universit, Power system and its automation, 2005.

4. Cheng jing-qing, Lin zhen-zhi, Liu qian-jin, et al. Relay protection setting management system based on $\mathrm{C} / \mathrm{S}$ and $\mathrm{B} / \mathrm{S}$ mode [J]. Power System Protection and Control, 2006,34(1): 18-21,57.

5. Lin tu-fang, Hong kai-xing, Guo cai-fu, et al. Implementation of on-line state monitoring system for transformer based on $\mathrm{B} / \mathrm{S}$ architecture $[\mathrm{J}]$. Journal Of Electronic Measurement And Instrumentation, 2013; 27(8): 766-772.

6. Liu he-ping, Luo hong-ping. The data acquisition and processing in transformer terminal unit[J]. Electrotechnical Application, 2006, 25(8):14-17.

7. Wei wen-xiang, Zhu zhi-jie. ATT7022 power/energy measurement IC with SPI and its application in monitoring of electric power terminal[J]. International Electronic Elements, 2005, (07):32-35.

8. Chou guo-qing, Yang zhi-long, Tang xian-lun, et al. The design and application of the watchdog circuit[J]. Automation and Instrumentation, 2005,(4):16-20.

9. Wei qiu-yue. The on-line monitoring system of electric parameter based on quasi-synchronous sampling[J]. Microcomputer Information, 2008, 24(19):301-303. 